\title{
Molecular and structural imaging in oncology, improvements and perfection "...the most effective multimodality approach and a significant advance for examining patients with cancer"
}

\author{
*Houseni, M. \& ** Alavi, A.
}

*MD, Department of Radiology, National Liver Institute, Menoufya University and Alfa scan, Egypt, ${ }^{* *} \mathrm{MD}, \mathrm{MD}$ (Hon), PhD (Hon), DSc (Hon), Department of Radiology, Hospital of the University of Pennsylvania, Philadelphia, PA. USA

Cancer is a major health problem and a leading cause of death in the world even if adequately treated. It will continue to be a chronic and debilitating disease. The key to improving the overall quality of life and to decrease lingering morbidity in cancer depends on continuous monitoring of disease activity, accurate staging, early detection of recurrence and initiation of appropriate and effective treatment.

Obviously, there are very complex undertakings in spite of major advances in recent years in medical imaging and other diagnostic initiatives. By now it has become clear that structural imaging with $\mathrm{CT}$ and MRI suffers from many shortcomings and cannot be relied upon solely for optimal management of these patients.

Positron emission tomography (PET) is a molecular imaging technology which utilizes tracers for assessing metabolic and biochemical pathways in a non-invasive and quantitative manner. By now, its role in oncology is well established by its ability to assess and characterize the metabolic and functional parameters of malignant tissues $[1,2]$. PET/CT and PET-MR fusion imaging is emerging as the most effective multimodality approach and a significant advance for examining patients with cancer.
The unique advantage of combined molecular and structural information provided by these multimodalities is to exploit the advantages of both and evaluate their role in many complex settings [3].

A large number of radiolabeled compounds targeting specific molecules or biochemical pathways have been and continue to be synthesized and validated as PET tracers. The leading tracer that is being widely used is $18 \mathrm{~F}$-fluorodeoxyglucose (FDG). The concept of the FDG technique was born in 1973 and was tested in 1976 at the University of Pennsylvania and ever since the critical role of molecular imaging in medicine emerged around the globe. This glucose analogue imitate glucose metabolism in the healthy and diseased tissue [4]. The idea behind using FDG in cancer is that the malignant cells are characterized by enhanced glucose consumption [5]; hence FDG-PET imaging allows detection and characterization of cancer based on the presence and the degree of 18F-FDG uptake. The evolution of FDGPET from a basic science and experimental undertaking to a powerful clinical modality has lead down a strong foundation for molecular imaging as a new specialty in medicine.

\section{Correspondence author:}

Mohamed Houseni, MD

Email: mohamedhouseni@gmail.com 
The use of PET/CT imaging in diagnostic radiology covers the fields of oncology, cardiology, neurology, and infection/ inflammation. However, the majority of PET/CT imaging is employed for examining oncology patients. This includes diagnosis, staging, restaging, detection of residual/recurrence disease, assessment of response to medical treatment and radiation therapy planning. A large number of publications proved evidence that PET/CT imaging has higher sensitivity and specificity than conventional imaging in a variety of cancers. This is particularly impressive in improving the specificity and to a great extent the sensitivity and overall accuracy over conventional techniques [6, 7]. A study by Hillner et al. [8] which assessed the impact of PET/CT imaging on patients' management showed altering conventional plans in $36.5 \%$ (95\% CI, 35.9 to 37.2) of cases after PET/CT imaging. In addition, PET can be utilized at different time points during the work-up of patients with cancer, as a complementary study to other imaging modalities, as a substitute for another procedure, or to seek information that cannot be provided by any other means.

By introduction of other positron emitting radiotracers, PET imaging is gaining newer applications in oncology. Targeted tracers span both diagnostic and therapeutic potential for newer treatment modalities. Several imaging strategies have been recently described for measuring selected properties of tumors including cell proliferation, over-expression of epidermal growth factor receptors, angiogenesis, hypoxia, apoptosis and the ability to invade and metastasize [9-15].

Several PET/MR prototype instruments have recently become a reality and offer several advantages in comparison to PET/CT. The assimilation of PET and MRI has been achieved without degrading the performance of the two techniques. The potential advantages of PET/MR include lack of additional radiation from $\mathrm{CT}$, better assessment of the brain, neck and musculoskeletal disorders and the utilization of MR related functional data including diffusion, perfusion, spectroscopy and elastography sequences $[16,17]$. Therefore, a hybrid PET/CT/MR instruments may become the optimal imaging platform in the future for combined both structural and functional imaging of cancer and other disorders. The main issue that poses the widespread ability of this approach is the attenuation correction of PET images by these combined systems.

Despite concerns related to the imaging cost and tracer availability, PET/CT imaging is steadily growing by demonstrating its effectiveness in eliminating unnecessarily invasive procedures and expensive surgical interventions. Moreover, based on the results of extensive publications, PET/CT imaging has become the most comprehensive oncology imaging modality at the present time.

\section{References}

1. Facey, K., et al., Overview of the clinical effectiveness of positron emission tomography imaging in selected cancers. Health Technol Assess, 11(44): p. iii-iv, xi267. 2007.

2. Basu, S. and A. Alavi, Unparalleled contribution of $18 F-F D G$ PET to medicine over 3 decades. J Nucl Med, 49(10): p. 17N21N, 37N. 2008.

3. Torigian, D.A., et al., Functional imaging of cancer with emphasis on molecular techniques. CA Cancer J Clin, 57(4): p. 20624. 2007.

4. Alavi, A. and M. Reivich, Guest editorial: the conception of FDG-PET imaging. Semin Nucl Med, 32(1): p. 2-5. 2002.

5. Warburg, O., K. Posener, and E. Negelein, The metabolism of the carcinoma cell. The Metabolism of Tumors. New York, Richard R. Smith, Inc, p. 129-169. 1931. 
6. Czernin, J., M. Allen-Auerbach, and H.R. Schelbert, Improvements in cancer staging with PET/CT: literature-based evidence as of September 2006. J Nucl Med, 48 Suppl 1: p. 78S-88S. 2007.

7. Czernin, J., M.R. Benz, and M.S. AllenAuerbach, PET/CT imaging: The incremental value of assessing the glucose metabolic phenotype and the structure of cancers in a single examination. Eur $\mathrm{J}$ Radiol, 73(3): p. 470-80. 2010.

8. Hillner, B.E., et al., The impact of positron emission tomography (PET) on expected management during cancer treatment: findings of the National Oncologic PET Registry. Cancer, 115(2): p. 410-8. 2009.

9. Bading, J.R. and A.F. Shields, Imaging of cell proliferation: status and prospects. $\mathrm{J}$ Nucl Med, 49 Suppl 2: p. 64S-80S. 2008.

10. Cai, W., G. Niu, and X. Chen, Multimodality imaging of the HER-kinase axis in cancer. Eur $\mathrm{J}$ Nucl Med Mol Imaging, 35(1): p. 186-208. 2008.

11. Dass, K., et al., Evolving role of uPA/uPAR system in human cancers. Cancer Treat Rev, 34(2): p. 122-36. 2008.
12. Jacobson, O., et al., 64Cu-AMD3100--a novel imaging agent for targeting chemokine receptor CXCR4. Bioorg Med Chem, 17(4): p. 1486-93. 2009.

13. Kenny, L.M., et al., Phase I trial of the positron-emitting Arg-Gly-Asp (RGD) peptide radioligand $18 \mathrm{~F}$-AH111585 in breast cancer patients. J Nucl Med, 49(6): p. 879-86. 2008.

14. Li, Z.B., et al., Imaging of urokinasetype plasminogen activator receptor expression using a 64Cu-labeled linear peptide antagonist by microPET. Clin Cancer Res, 14(15): p. 4758-66. 2008.

15. Zannetti, A., et al., Imaging of alpha(v)beta(3) expression by a bifunctional chimeric RGD peptide not cross-reacting with alpha(v)beta(5). Clin Cancer Res, 15(16): p. 5224-33. 2009.

16. Pichler, B.J., M.S. Judenhofer, and H.F. Wehrl, PET/MRI hybrid imaging: devices and initial results. Eur Radiol, 18(6): p. 1077-86 2008.

17. Katz, S., et al., PET, $C T$, and $M R$ Imaging for Assessment of Thoracic Malignancy: Structure Meets Function. PET Clinics, 3(3): p. 395-410. 2008. 